\title{
$50+$ years of replantation surgery experience: are we progressing or regressing?
}

\author{
Karen Noh, Jacques H. Hacquebord \\ Department of Orthopaedic Surgery, NYU Langone Health, New York, NY 10014, USA.
}

Correspondence to: Dr. Jacques H. Hacquebord, Department of Orthopaedic Surgery, NYU Langone Health, 530 1st Avenue, New York, NY 10016, USA. E-mail: Jacques.Hacquebord@nyulangone.org

How to cite this article: Noh K, Hacquebord JH. 50+ years of replantation surgery experience: are we progressing or regressing? Plast Aesthet Res 2020;7:50. http://dx.doi.org/10.20517/2347-9264.2020.49

Received: 15 Mar 2020 First Decision: 12 Aug 2020 Revised: 21 Aug 2020 Accepted: 4 Sep 2020 Published: 17 Sep 2020

Academic Editor: Alessandro Thione Copy Editor: Cai-Hong Wang Production Editor: Jing Yu

\begin{abstract}
The first successful digit replantation was reported in 1965 and accepted enthusiastically by hand surgeons. The decade that immediately followed saw a surge of interest in this complex surgery, fueling significant improvements in success rates and the rise of hand and microsurgeons who were highly proficient in replantation. The decades that followed, however, showed a stable field lacking any significant changes or advancements. More recently, and especially in the United States, the frequency with which surgeons even attempt replantation and the rate of survival have plummeted. If this trend continues, successful replantation surgery will become all too rare of an event. It is critical that we evaluate the state of replantation surgery today, identify the primary causes, and work to not only revive the field but allow it to advance similar to other areas of medicine.
\end{abstract}

Keywords: Digit, replantation, amputation, hand surgery, microsurgery

\section{INTRODUCTION}

In $2017,14.7 \%$ of primary diagnoses at emergency department visits in the United States were classified as an injury to the wrist, hand, and fingers ${ }^{[1]}$. Injuries to the hand and digits are exceedingly common - of these, traumatic amputations of the digit are among the most severe injuries possible. The hand surgeon is faced with two important options in treating this devastating injury: replantation or revision amputation.

Studies have demonstrated that replantation may be more desirable: a 2019 study reported significantly better functional outcomes in patients with successful replantation than revision outcomes, as measured

CC (i) (c) The Author(s) 2020. Open Access This article is licensed under a Creative Commons Attribution 4.0 EY International License (https://creativecommons.org/licenses/by/4.0/), which permits unrestricted use sharing, adaptation, distribution and reproduction in any medium or format, for any purpose, even commercially, as long as you give appropriate credit to the original author(s) and the source, provide a link to the Creative Commons license, and indicate if changes were made.

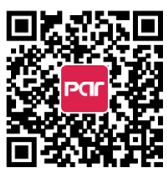


by nine-hole peg test times, Semmes-Weinstein monofilament test, and three-point pinch test. Patientreported outcomes - the Michigan Hand Outcomes Questionnaire; Disabilities of the Arm, Shoulder, and Hand; and Patient-Reported Outcomes Measurement Information System upper-extremity module scores and functional outcomes - were significantly higher as well ${ }^{[2]}$. While successful replantation appears to be superior to revision amputation, there has been an alarming decrease in attempts to replant amputated digits.

We start with the history of digit replantation to better understand the current state of digit replantation and how our techniques have evolved - or not evolved.

\section{EARLY HISTORY}

In 1894, Sadi Carnot, the president of France, was assassinated after a stab wound to the abdomen that lacerated his portal vein. Surgeons of the time claimed he could not have been saved due to the nature of his injuries. Alexis Carrel, then a young medical student at the University of Lyon, disagreed ${ }^{[3]}$. He began experimenting, and by 1902 he had published his first articles on vascular anastomosis ${ }^{[4]}$.

Carrel performed the first extremity replantation in 1906, the amputated hind limb of a dog ${ }^{[5]}$. Much of his work became pillars of organ transplantation and heart surgery, and he later became the recipient of the Nobel Prize in Physiology or Medicine for his contributions to vascular suture and transplantation of blood vessels and organs ${ }^{[6]}$. These early developments in vascular anastomosis made replantation of limbs, and eventually digits, a possibility.

\section{EARLY BARRIERS TO SUCCESSFUL REPLANTATION}

There exists a nearly 60-year gap between Carrel's early experimentation with limb replantation in canines in 1907 and Ronald Malt's first limb replantation in a human in $1964^{[7]}$. This gap was at least in part due to the lack of appropriate equipment and technology. Antibiotics were not discovered until the late 1920 s and were not standard postoperative protocol until even later. Anticoagulants such as heparin and coumadin were not discovered until 1936 and $1941^{[5]}$. The operating microscope was introduced in 1921, but it was not used for microvascular surgery until $1960^{[8]}$. Finally, Harry Buncke developed the first set of microsurgical instruments including microsutures and microsurgical needles ${ }^{[9]}$.

With these developments, the major technical challenges to digital replantation had been addressed: proper suture technique to minimize the risk of hemorrhage, stenosis, and thrombosis; antibiotics to prevent infection; anticoagulants to prevent clotting; the operating microscope for better visualization; and the appropriate tools for microvascular surgery. The stage was set.

\section{THE FIRST SUCCESSFUL DIGIT REPLANTATION}

In 1965, Komatsu and Tamai ${ }^{[10]}$ reported the first successful digital replantation. They had attempted several replantations of completely amputated digits over a year, none of which were successful. In a 4.5-h surgery, Komatsu and Tamai replanted a completely amputated thumb, performing an end-to-end anastomosis on two volar digital arteries and two volar dorsal veins. The patient was discharged 40 days after the landmark surgery, returning to his original occupation only four months after his injury. Remarkably, 200 days after the operation, the patient displayed only slight atrophy and loss of sensation. The case report was published in 1968 , and it was received enthusiastically by the medical community.

Such great success of the world's first reported digit replantation begs the question: How much has improved since then? Is there more to be improved, or have we already reached the limits of success in digit replantation? 


\section{EARLY CHALLENGES}

Following the first reported successful replantation, there was a surge of interest in replantation surgery ${ }^{[11]}$. Hand/microsurgeons attempted replantation with enthusiasm. However, replanted digits often lacked function and sensation; some were even painful ${ }^{[11]}$. Reattaching the amputated digit simply because it was possible was not enough.

As it became clear that digit amputations were not all equal, hand surgeons first looked to case selection as a means of improving success rates. The first consideration was if the digit was in suitable condition for replantation. For instance, a severe crush injury causing comminuted fractures and significant soft tissue disruption may not be eligible for replantation. Fingers that were stored improperly during transport, such as in non-biologic solutions or on dry ice, were definite contraindications.

In 1978, a replantation team in Vienna compiled their three-year experience in replantation, which included a set of indications ${ }^{[12]}$. They stated replantation should always be attempted in any amputations in young patients, especially children. Thumbs should always be replanted but not the other fingers except in the case of multiple digit amputations causing loss of the ability to grip. They believed single finger amputations should be performed only if the patient required that digit for their profession, skills, or hobbies. In 1981, Dr. Zhong-Wei, Dr. Meyer, Dr. Kleinert, and Dr. Beasley, today regarded as pioneers in replantation, together compiled the experiences from the authors' home institutions in China, Switzerland, and the United States ${ }^{[13]}$. While they admit that firm indications for finger replantation are impossible, their experiences largely matched those published by the Viennese replantation team. Since then, the indications and contraindications have mostly remained unchanged ${ }^{[11,14,15]}$ :

1. Thumb amputations

As the thumb is responsible for up to $40 \%$ of hand function, all thumb amputations should be considered for replantation. Factors that may constitute contraindications in any other single digit amputation are underemphasized relative to the importance of the thumb ${ }^{[16]}$.

2. Multiple digit amputations

Unsurprisingly, the more digits that are successfully replanted, the greater is the final function. Replantation is attempted starting with the digit with the greatest contribution to hand function and greatest chance of recovery ${ }^{[17]}$.

3. Mid-palm amputations

Amputations at the mid-palm level or more proximally are replanted more successfully than amputations at the level of the digital arteries. Following successful replantations at this level, function is far superior to any prosthetics ${ }^{[18]}$.

4. Single digit amputation distal to FDS tendon insertion

Reported as early as 1981 by Dr. Zhong-Wei, amputations distal to insertion of the FDS tendon were found to have superior outcomes ${ }^{[13]}$. Replanting digits proximal to the insertion of the FDS tendon often results in a stiff proximal interphalangeal joint.

5. Amputations in pediatric patients

Pediatric patients have superior healing potential compared to adults. However, their anatomy is even smaller than that of adult patients and thus cases in pediatric patients may be even more challenging.

While these guidelines were a good place to start, the exact details of the injury and the patient were determined to be equally, if not more, important. A detailed history - including the circumstances of the injury, past medical history, and social history were found to be critical in order to determine if replantation was worthwhile. Mechanism was also found to be a crucial component to the consideration that directly affects the zone of injury and likelihood for a successful outcome.

While these guidelines were a good place to start, the exact details of the injury and the patient were determined to be equally, if not more, important. A detailed history - including the circumstances of the 
injury, past medical history, and social history were found to be critical in order to determine if replantation was worthwhile. Mechanism was also found to be a crucial component to the consideration that directly affects the zone of injury and likelihood for a successful outcome. A final consideration is ischemia time, especially in rural or medically underserved areas where a patient may need to travel significant distances or be transferred to a trauma center with microsurgical service availability. Traditionally, it has been taught that prolonged ischemia time negatively impacts success rates. However, recent literature has shown that ischemia time may not play a large role in digit replantation success. A meta-analysis found ischemia time failed to influence replant survival ${ }^{[19]}$. This is likely because digits do not have large muscle mass, and therefore are less vulnerable to rapid necrosis. However, reperfusion injury remains a significant risk and urgent, although perhaps not immediate, replantation is necessary. Current guidelines suggest limits of $12 \mathrm{~h}$ of warm ischemia time or $24 \mathrm{~h}$ of cold ischemia time, although this has increasingly been challenged ${ }^{[20]}$. Lin and colleagues reported a success rate of $64.0 \%$ in a small cohort of 14 patients who underwent replantation after $24 \mathrm{~h}$ of ischemia ${ }^{[21]}$. In as early as 1988 , Wei and colleagues reported successful replantations after 84 , 86 , and 94 h of cold ischemia time ${ }^{[22]}$.

An interesting addendum to extending ischemia time is cryopreservation. A critical component of successfully reattaching a cryopreserved part is uniform freezing and thawing; because digits do not have significant muscle mass, they have potential to be successfully replanted following cryopreservation. Wang and colleagues reported two successful cases of digit replantation after cryopreservation - one left index finger cryopreserved in liquid nitrogen for 10 days before replantation, and one left thumb cryopreserved in liquid nitrogen for 30 days before replantation ${ }^{[23]}$.

Surgeons from early on appreciated how comorbidities were an important consideration, as they may influence vasculature quality. Hustedt and colleagues found that the rates of replant failure were highest in patients with psychotic disorders, peripheral vascular disease, and electrolyte imbalances ${ }^{[2]}$. As significant medical comorbidities were of concern, age quickly became a relative contraindication. Concern was certainly warranted in older patients as with any long and invasive surgery. However, Kwon and colleagues found that microsurgical success and satisfaction with the results of the procedure were comparable in elderly patients (70 years and older) versus younger patients ${ }^{[25]}$.

From early on, it was realized that many contraindications and indications for replantation were not absolute. Surgeons had the responsibility to carefully weigh the risks and benefits of a variety of factors which included more than just the medical facts. As Dr. Zhong-Wei said ${ }^{[13]}$, “The psychological response to injury, the importance of body image, and the concern for disfigurement vary enormously among cultures but in many may be as important as the restriction of physical abilities." He noted that, in some cultures, an intact but nonfunctional digit is of greater value than an absent digit that is functionally compensated for by other digits or a prosthetic, and vice versa in other cultures. He concluded that "function" cannot be measured solely by physiology and ability, but rather it must also embrace the patient's very personal perception of a successful outcome.

\section{REPLANTATION TODAY}

Success rates of replantation rapidly increased as surgeons gained experience and selected cases more prudently, with one study reporting a survival rate of $97 \%$ in as early as $1985^{[26]}$. However, the recent literature is less optimistic.

A recent meta-analysis compiled the results of 32 studies with comparable inclusion criteria and outcome measures to determine factors associated with increased survival ${ }^{[19]}$. An interesting trend emerged: during 1985-2015, survival attempts of studies done in the US ranged 48\%-86\%, while rates those coming from Asia, namely China, Japan, Korea, Taiwan, and India, ranged 68\%-97\%. While survival rates in Asian 


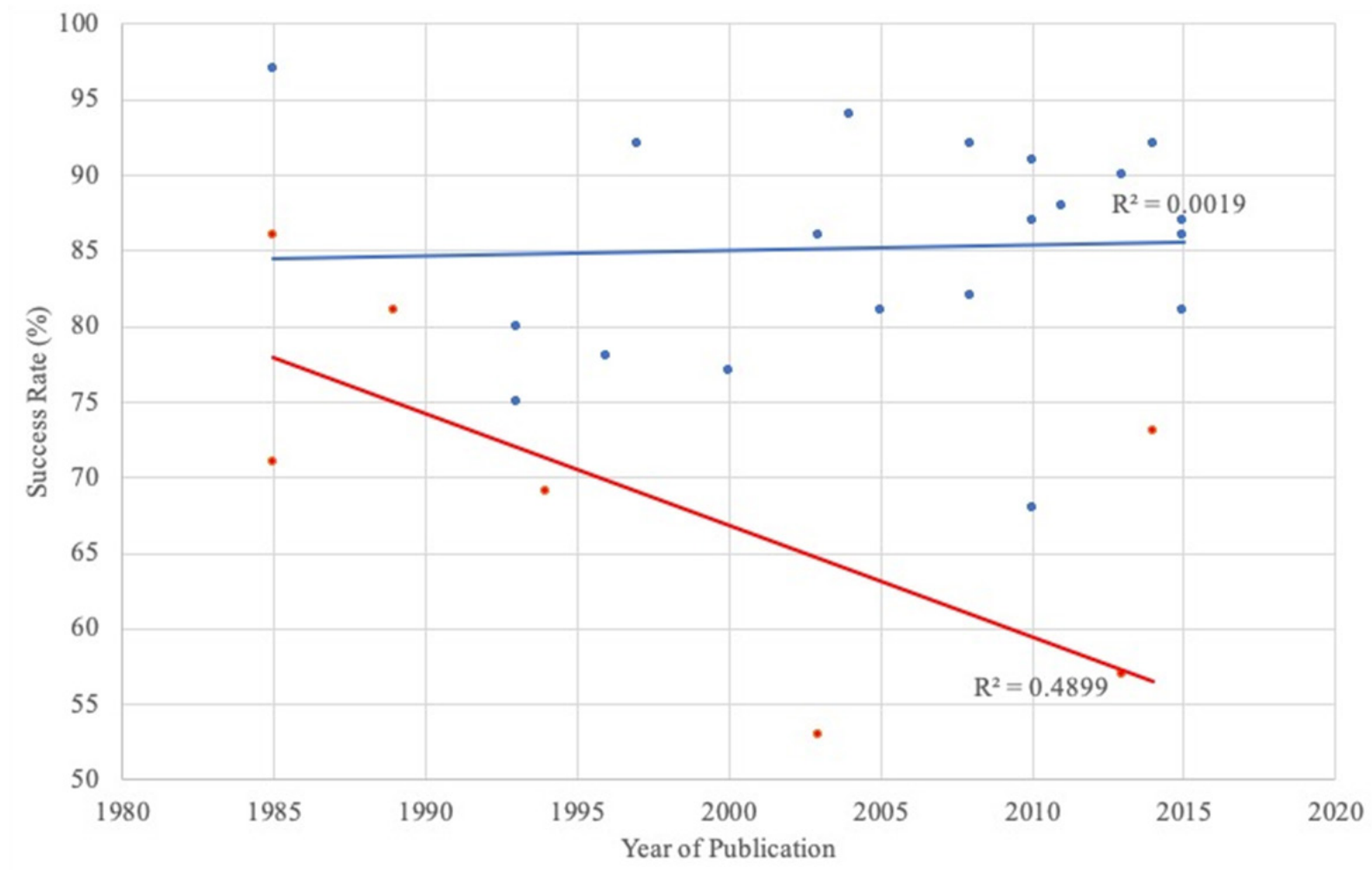

Figure 1. The success rates of 28 studies in Asia versus the United States. We plotted the success rates reported in 28 studies, derived from the 2018 meta-analysis by Shaterian and colleagues. Linear regression in this case is not intended to suggest mathematically or statistically meaningful trends, but it demonstrates a simplified visual of the trend of success rates in Asian countries (China, Japan, Korea, Taiwan, and India) versus the United States

countries largely remained constant, survival rates in the US have plummeted [Figure 1]. In 2013, Fufa and colleagues reported only a 57\% success rate for replantation of Tamai Level III or IV amputations performed at two large academic Level I hospitals ${ }^{[27]}$. Mulders and colleagues reported a success rate of $48 \%{ }^{[28]}$. A large academic replantation center in 2019 reported a survival rate of only $50 \%{ }^{[29]}$. This is in stark contrast to success rates in Asian countries. What is the cause of this discrepancy? More importantly, how can we improve?

\section{IN THE LITERATURE: UNCERTAINTY AND DISAGREEMENT}

The field of replantation developed significantly in the early days, but this trend has not continued through the decades. There remains great variability in surgeons' preferred techniques in nearly all aspects of the treatment. The pioneers in replantation anticipated that survival rates would increase as techniques advanced. However, the literature seems to show more disagreement and a lack of definitive answers than advancements in techniques and innovation.

Even the sequence of steps for when vascular anastomoses is performed varies between surgeons. In Green's Operative Hand Surgery, the recommended sequence of repair is: osseous fixation, extensor tendons, flexor tendons, digital nerves, dorsal veins, and arteries ${ }^{[30]}$. Chung and Alderman, however, recommended the artery before the vein ${ }^{[31]}$. Anecdotally, high volume replantation surgeons will proceed only if good veins can be identified. Other surgeons have argued that venous outflow is not essential for successful surgery ${ }^{[29]}$. While these distinctions may seem minor, survival of the digit is solely dependent on establishing adequate arterial inflow and venous outflow. 
The optimal osseous fixation remains debated. Lee et al. ${ }^{[32]}$ retrospectively evaluated 103 replanted digits. Of their 32 digits with radiographic nonunion, the highest rate of nonunion was found after cross fixation and the lowest after intraosseous wire alone; however, this difference was not statistically significant. Hoffman and colleagues concluded that intraosseous wire was better for fracture fixation and early hand therapy as well $^{[33]}$. However, Cheng et al. ${ }^{[34]}$ concluded that rigid internal fixation with plate and screws were superior in their cohort. Anecdotally, k-wire fixation remains one of the most common techniques seen with many replantation surgeons. Further prospective studies are needed to determine the optimal method of bony fixation which minimizes complications while maximizing the possibility for early motion and successful replantation.

Uncertainty regarding the use of postoperative anticoagulants was reported as early as 1978, and remains an area of disagreement today ${ }^{[35]}$. Anticoagulants are used widely following replantation surgery. However, the appropriate type, dose, and timing between surgeons and institutions varies widely, ranging from no thromboprophylaxis at all to various doses of aspirin, dextran, and heparin ${ }^{[36]}$. Even the specifics of route, quantity, and what type of heparin is administered varies greatly among surgeons. A 2019 randomized and single-blinded study found no significant difference between the three groups: (1) no heparin; (2) low dose heparin (10,000 IU/day); and (3) high dose heparin (starting at 15,000 IU/day and adjusted to achieve activated partial thromboplastin time of 1.5-2.5 times the patient's baseline $)^{[37]}$. Studies with various therapeutic evidence levels have supported these conclusions ${ }^{[38,39]}$. In addition to dose, one study found increased survival with progressive weaning of heparin rather than abruptly discontinuing $i t^{[40]}$. While systematic heparinization does not appear to impact success rates, two recent studies have supported digit salvage using local injections of heparin ${ }^{[41]}$ or heparin calcium ${ }^{[42]}$. Preventing complications with arterial inflow and venous outflow is critical for obvious reasons and also because even if caught early salvage rates remain low ${ }^{[43]}$. Clearly, further research is needed to clarify the specific type, dose, and timing that is most effective.

As reflected in the literature, there remains significant disagreement about which techniques and interventions result in the highest success rates. More and higher quality research is urgently needed to answer pressing questions about each step of replantation surgery.

\section{IN PRACTICE: DWINDLING INTEREST AND ATTEMPTS}

Discouraging rates of success in combination with the lack of consensus on the best techniques and postoperative protocols have likely contributed to decreasing interest and attempts at replantation surgery in the West. Some have suggested that this decrease in attempts is natural, caused by a decrease in industrial accidents. A 2018 study found, however, that, while workplace finger amputations decreased significantly between 2000 and 2010 in the US, overall finger amputation incidence did not change significantly. However, in that same period, replantation surgery decreased by more than $50 \%{ }^{[4]}$. The conclusion is that attempts at replantation in the US have decreased despite consistent incidence of amputations. There are likely several factors that have contributed to this.

Peterson and colleagues found inconsistent availability of hand/microsurgeons at Level I and II trauma centers in the US, leading to inadequate evaluation of the amputated digit, and decreased likelihood that replantation would even be considered ${ }^{[45]}$. Related to this, Hustedt and colleagues theorized that decreasing success rates in the US are correlated with decentralization of replants away from high-volume hospitals ${ }^{[46]}$. They found that high-volume surgeons (more than five replants per year) at high-volume hospitals (more than 20 replants per year) had greater success rates than low-volume surgeons at low-volume hospitals $92.0 \%$ compared to $72.1 \%$. Their proposed solution was centralization of replantations. In distinction to this, Cho et al. ${ }^{[2]}$ found that "neither hospital case volume nor hospital type was predictive of successful replantation". Replantation attempts decreased at all hospital types: rural or urban, teaching or non- 
teaching. They concluded that centralization alone would not be effective but that other factors required to be addressed.

An interesting finding in the Cho et al. ${ }^{[2]}$ study was that patients with private insurance were twice as likely to receive replantation in comparison to patients with Medicare or Medicaid. As payer status directly influences reimbursement, they suggested that financial incentive may play a role. This is further supported by a recent analysis of reimbursement information for 51,716 patients by Hooper and colleagues, who determined that replantation reimburses at $\$ 78 / \mathrm{wRVU}$, which is significantly lower compared to revision amputation (\$108) or common procedures such as carpal tunnel release (\$101), trigger finger release $(\$ 116)$, and extensor tendon repair $(\$ 122)^{[47]}$. Physician work relative value units (wRVU) is a direct measure of physician reimbursement in the United States and, in this case, an indirect indicator of the perceived value of a procedure. For both surgeons and payers, a common misconception is that there is minimal value in replantations. This is in stark contrast to the literature, which shows good functional outcomes and high patient satisfaction ${ }^{[15,48]}$.

It has been proposed that surgeon experience with microsurgery and anastomoses of fine veins is more important to successful replantation than surgical technique ${ }^{[11]}$. A conclusion of the above evidence is that the stagnant or even decreasing survival rates result from a lack of experience. In the US, this has unfortunately led to a self-perpetuating cycle: lack of experience leads to decreasing success rates, which leads to decreased confidence and incentive, fewer attempts, and thus further lack of experience.

\section{CONCLUSION}

The current literature reveals two problems. First, there is a stagnation of the techniques and knowledge associated with replantation in the literature. There are few conclusive statements that can be made of the intricacies of the surgery. Second, and more discouraging, there is a regression of the field in the Western hemisphere, most notably in the US. The rates of replantation and survival are both decreasing - this is evidence of regression of medical care in the US. These two problems paint a concerning picture for the current state of replantation surgery.

In 21 st century medicine, we expect a continual and forward march in our knowledge, innovation, treatments, and solutions. The current state of replantation surgery is unfortunately not consistent with this. Can or should we expect any better in the future? If so, there are very important changes that need to take place. Below are four important points that, if appropriately implemented, can allow for progression in replantation surgery.

(1) Further research is urgently needed to better understand the barriers of successful replantation, specifically in the United States. The current literature on replantation varies widely. Length of follow up, how function is determined, and patient-reported outcomes vary from study to study. A coordinated effort with consistent measures of function and patient-reported outcomes, similar to the 1981 report by Dr. Zhong-Wei and other leaders in replantation, would be immensely valuable. Since injury patterns and techniques are heterogenous, well designed and large prospective multi-center studies are a necessary part of the solution.

(2) The evidence clearly supports the importance of technical skill, frequency of replantation, and clinical experience as critical for improved survival rates. It is no secret that there are centers with much higher success rates. Centralizing replantation surgery, especially in the United States, is an essential component of this and naturally fosters the formation for centers of excellence. The knowledge and skill gained at these centers of excellence must be shared and taught to younger replantation surgeons allowing for elevation of the entire field. 
(3) Successful replantation relies on a team of people that work in concert. From the operating theatre, to the hospital ward, the therapists' suite, and the doctor's office-all members of the team must be knowledgeable, dedicated, and skilled at treating this unique patient population. Only with this team in place should replantation be embarked. The surgery is only one component of the patient's care.

(4) Replantation surgery is a unique surgical procedure and involves the treatment of multiple different tissue structures: osseous, nerve, skin, vasculature, and connective tissue. Survival of the digit focuses primarily on only one tissue structure - the vasculature. However, successful replantation, as discussed above, must include consideration of function. For this reason, equal attention must be placed on treating all tissue structures. When one tissue structure is either prioritized or neglected compared to the others, the function will suffer.

The future of replantation surgery is at a crossroads. If current trends in the United States remain, successful replantation will become an uncommon occurrence with ever increasing contraindications. As the advancements in partial hand and finger prosthetics have accelerated, some may begin to argue that digit replantation is never necessary. Whether or not the technology will ever develop to the point of being equal to a native and well-functioning digit is uncertain and debatable. Irrespective, technology is far from that point yet and our patients are still in need of successful replantation surgery. Especially in the United States, we must acknowledge that the state of replantation surgery has regressed and is inferior to some of our colleagues around the world. The field of hand surgery and our patients require that we not accept the current state. We must make the necessary changes to further the current standard.

\section{DECLARATIONS}

\section{Authors' contributions}

Made substantial contributions to review of literature, writing of article: Noh K, Hacquebord JH

\section{Availability of data and materials}

Not applicable.

\section{Financial support and sponsorship}

None.

\section{Conflicts of interest}

Both authors declared that there are no conflicts of interest.

\section{Ethical approval and consent to participate}

Not applicable.

\section{Consent for publication}

Not applicable.

\section{Copyright}

(c) The Author(s) 2020.

\section{REFERENCES}

1. Rui P, Kang K. National hospital ambulatory medical care survey: 2017 emergency department summary tables. National Center for Health Statistics. Available from: https://www.cdc.gov/nchs/data/nhamcs/web_tables/2017_ed_web_tables-508.pdg. [Last accessed on 10 Sep 2020]

2. Cho HE, Zhong L, Kotsis SV, Chung KC. Finger replantation optimization study (FRONT): update on national trends. J Hand Surg Am 
2018;43:903-12.e1.

3. Rothwell A. Alexis Carrel: innovator extraordinaire. J Perioper Pract 2011;21:73-6.

4. Dente CJ, Feliciano DV. Alexis Carrel (1873-1944): Nobel laureate, 1912. Arch Surg 2005;140:609-10.

5. Kocher MS. History of replantation: from miracle to microsurgery. World J Surg 1995;19:462-7.

6. The Nobel Prize. The nobel prize in physiology or medicine 1912: Alexis Carrel. Available from: https://www.nobelprize.org/prizes/ medicine/1912/summary/. [Last accessed on 15 Mar 2020]

7. Malt R, McKhann CF. Replantation of severed arms. JAMA 1964;189:716-22.

8. Kriss TC, Kriss VM. History of the operating microscope: from magnifying glass to microneurosurgery. Neurosurgery 1998;42:899-908.

9. American Association of Plastic Surgeons. Memoirs: Harry J. Buncke, M.D. 1922-2008. Available from: https://aaps1921.org/memoirs/ HarryBunkle.cgi. [Last accessed on 15 Mar 2020]

10. Komatsu S, Tamai S. Successful replantation of a completely cut-off thumb. Plast Reconstr Surg 1968;42:374-7.

11. Hadley SR, Capo JT. Digit replantation the first 50 years. Bull Hosp Jt Dis 2013;73:148-55.

12. Berger A, Millesi H, Mandl H, Freilinger G. Replantation and revascularization of amputated parts of extremities: a three-year report from the Viennese replantation team. Clin Orthop Relat Res 1978;133:212-4.

13. Zhong-Wei C, Meyer VE, Kleinerg HE, Beasley RW. Present indications and contraindications for replantation as reflected by long-term functional results. Orthop Clin North Am 1981;12:849-70.

14. MacLoed AM, O’Brien BM, Morrison WA. Digital replantation: clinical experiences. Clin Orthop Relat Res 1978;133:26-34.

15. Pet MA, Morrison SD, Mack JS, Sears ED, Wright T, et al. Comparison of patient-reported outcomes after traumatic upper extremity amputation: Replantation versus prosthetic rehabilitation. Injury 2016;47:2783-8.

16. Soucacos PN, Beris AE, Malizos KN, Touliatos AS. Bilateral thumb amputation. J Hand Surg Am 1982;7:549-56.

17. Salah MM, Khalid KN. Replantation of multiple digits and hand amputations: four case reports. Cases J 2008;1:266.

18. Beris AE. Lykissa MG, Korompilias AV, Mitsionis, GI. Vekris MD, et al. Digit and hand replantation. Arch Orthop Trauma Surg 2010;130:1141-7.

19. Shaterian A, Rajaii R, Kanack M, Evans G, Leis A. Predictors of digit survival following replantation: quantitative review and metaanalysis. J Hand Microsurg 2018;10:66-73.

20. Wolfe VM, Angela AW. Replantation of the upper extremity: current concepts. J Am Acad Orthop Surg 2015;23:373-81.

21. Lin CH, Aydyn N, Lin YT, Hsu CT, Lin CH, et al. Hand and finger replantation after protracted ischemia (more than 24 hours). Ann Plast Surg 2010;64:286-90.

22. Wei FC, Chang YL, Chen HC, Chuang CC. Three successful digital replantations in a patient after 84, 86, and 94 hours of cold ischemia time. Plast Reconstr Surg 1988;82:346-50.

23. Wang J, Lin J, Pei Y, Xu Q, Zhu, L. Crypreservation and transplantation of amputated finger. J Cryobiol 2020;92:235-40.

24. Hustedt JW, Chung A, Bohl DD, Olmscheid N, Edwards S. Evaluating the effect of comorbidities on the success, risk, and cost of digital replantation. J Hand Surg Am 2016;41:1145-52.e1.

25. Kwon GD, Ahn JS, Park YG, Chang GW, Ha YC. The effect of patient age on the success rate of digital replantation. Plast Reconstr Surg 2017;139:420-6.

26. Cheng GL, Pan DD, Yang ZX, Qu ZY. Replantation of digits amputated at or about the distal interphalangeal joint. Ann Plast Surg 1985;15:465-73.

27. Fufa D, Calfee R, Wall L, Zeng W, Goldfarb C. Digit replantation: experience of two U.S. academic level-I trauma centers. J Bone Joint Surg Am 2013;95:2127-34.

28. Mulders MAM, Neuhaus V, Becker SJE, Lee SG, Ring DC. Replantation and Revascularization vs. Amputation in Injured Digits. Hand 2013;8:267-73.

29. Milone MT, Klifto CS, Lee ZH, Thanik V, Hacquebord JH. Relationships between vein repairs, postoperative transfusions, and survival in single digit replantation. Hand (N Y) 2020;15:488-94.

30. Wolfe SW, Hotchkiss RN, Pederson WC, Kozin SH, Cohen MS, et al. Replantation. In: Green's operative hand surgery. 7th ed. Philadelphia: Elsevier Health Scicnces; 2016. pp. 1476-85.

31. Chung K, Alderman AK. Replantation of the upper extremity: indications and outcomes. J Hand Surg Am 2002;2:78-94.

32. Lee SW, Lee DC, Kim JS, Roh SY, Lee KJ. Analysis of bone fixation methods in digital replantation. Arch Plast Surg 2017;44:53-8.

33. Hoffmann R, Buck-Gramcko D. Osteosynthesis in digital replantation surgery. Ann Chir Gynaecol 1982;71:14-8.

34. Cheng HS, Wong LY, Chiang LF, Chan I, Yip TH, et al. Comparison of methods of skeletal fixation for severely injured digits. Hand Surg 2004;9:63-9.

35. Phelps D, Lilla JA, Boswick JA. Common problems in clinical replantation and revascularization in the upper extremity. Clin Orthop Relat Res 1978; 133:11-25.

36. Buckley T, Hammert WC. Anticoagulation following digital replantation. J Hand Surg Am 2011;36:1374-6.

37. Nishijima A, Yamamoto N, Gosho M, Yanagibayashi S, Yoshida R. Appropriate use of intravenous unfractionated heparin after digital replantation: a randomized controlled trial involving three groups. Plast Reconstr Surg 2019;143:1224e-32e.

38. Chen YC, Chi CC, Chan FC, Wen YW. Low molecular weight heparin for prevention of microvascular occlusion in digital replantation. Cochrane Database Syst Rev 2013;7:CD009894.

39. Retrouvey H, Solaja O, Batlzer HL. Role of postoperative anticoagulation in predicting digit replantation and revascularization failure: a propensity-matched cohort study. Ann Plast Surg 2019;83:542-7.

40. Efanov JI. Khriguian J, Cassier S, Boghossian E, Harris PG, et al. Duration and cessation characteristics of heparinization after finger 
replantation: A retrospective analysis of outcomes. Microsurgery 2018;38:251-8.

41. Alfesky H, McArthur P, Helmy Y. Salvaging digital replantation and revascularisation: efficiency of heparin solution subcutaneous injection. Surg Res Pract 2018;2018:1601738.

42. Kadota H, Imaizumi A, Ishida K, Sashida Y. Successful local use of heparin calcium for congested fingertip replants. Arch Plast Surg 2020;47:54-61.

43. Tejedor Navarro A, Vendrell Jordà M, Puente Alonso C. Digital replantation/revascularization: predictive factors to microsurgery success-a single-center study. Eur J Trauma Emerg Surg 2019; doi: 10.1007/s00068-019-01226-X.

44. Reavey PL, Stranix JT, Muresan H, Soares M, Thanik V. Disappearing digits: analysis of national trends in amputation and replantation in the united states. Plast Reconstr Surg 2018;141:857e-67e.

45. Peterson BC, Mangiapani D, Kellog R, Leversedge FJ. Hand and microvascular replantation call availability study: a national real-time survey of Level-I and Level-II Trauma Centers. J Bone Joint Surg Am 2012;94:e185.

46. Hustedt JW, Bohl DD, Champagne L. The detrimental effect of decentralization in digital replantation in the United States: 15 Years of evidence from the national inpatient sample. J Hand Surg Am 2016;41:593-601.

47. Hooper RC, Sterbenz JM, Zhong L, Chung KC. An in-depth review of physician reimbursement for digit and thumb replantation. J Hand Surg Am 2019;44:443-53.

48. Sebastin SJ, Chung KC. A systematic review of the outcomes of replantation of distal digital amputation. Plast Reconstr Surg 2011;128:723-37. 